\title{
Automated Preoperative Planning of Femoral Component for Total Hip Arthroplasty (THA) from 3D CT Images*
}

\author{
Itaru OTOMARU**, Masahiko NAKAMOTO***, Masaki TAKAO***, \\ Nobuhiko SUGANO***, Yoshiyuki KAGIYAMA ${ }^{\dagger}$, Hideki YOSHIKAWA ${ }^{* * *}$, \\ Yukio TADA** and Yoshinobu SATO*** \\ ** Graduate School of Engineering, Kobe University \\ 1-1 Rokkodai, Nada, Kobe, Hyogo 658-0003, Japan \\ *** Graduate School of Medicine, Osaka University \\ 2-2 Yamadaoka, Suita, Osaka 565-0871, Japan \\ $\dagger$ The Center for Advanced Medical Engineering and Informatics, Osaka University \\ 2-2 Yamadaoka, Suita, Osaka 565-0871, Japan \\ E-mail: otomaru@opt.cs.kobe-u.ac.jp
}

\begin{abstract}
The present paper describes a method for 3-dimensional (3D) automated preoperative planning of the femoral component (stem) in total hip arthroplasty (THA). Stem planning is formulated as a problem to determine the optimal parameters of position, rotation, and stem size, based on 3D surface models of host bone reconstructed from CT images, which includes the femur and femoral canal. An objective function that represents the fitness between the femoral canal and stem surfaces is defined. We also defined positional and rotational constraints derived from previous studies on femoral anatomy. Maximization is performed by the Powell method using initial values equally sampled within the possible solution space. We obtained parameters that maximize the objective function by exhaustive adjustment of the parameters. We applied the proposed method to 17 cases, and the proposed method was experimentally evaluated according to differences between planning results of the automated system and those of an experienced surgeon. Preoperative planning was also performed by a surgeon to evaluate performance of the automated system. The difference in stem size was less than 1 size in all cases, and the surgeon agreed with the planning results of the proposed method in 14 cases. In those 14 cases, mean errors of position and orientation were $3.2 \mathrm{~mm}$ and $4.6 \mathrm{deg}$, respectively. The proposed method thus appears applicable in preoperative planning of the stem.
\end{abstract}

Key words : Total Hip Arthroplasty, Computer-Assisted Surgery, Automated Surgical Planning System, Orthopedic Implant, Femoral Component, Stem Fitness, Anteversion Angle

\section{Introduction}

Recently, surgical navigation and robotic systems have been developed for total hip arthroplasty (THA) to allow accurate placement of orthopedic implants in preoperatively determined 3-dimensional (3D) position and orientation ${ }^{(1)}$ (7). Preoperative planning is thus becoming increasingly important because the preoperative plan can be accurately devised using these systems. In preoperative planning of THA, anatomical compatibility of each implant component with the host bone is essential for stable fixation and good clinical results. Recently, computed tomography (CT)-based interactive systems for preoperative 3D planning have been developed to quantitatively visualize the stable fixation. ${ }^{(8),(9)}$. However, interactive 3D planning is subjective and time-consuming, limiting clinical use. 
To solve these problems, we have been developing an automated preoperative planning system for THA, to automatically select the optimal size, position, and orientation of each implant. This system calculates the plan of the pelvic implant (cup) and femoral implant (stem) independently ${ }^{(10)-(12)}$, then integrates these intermediate solutions considering the difference in limb length and range of motion (ROM) of the hip joint ${ }^{(13)}$. To develop the automated planning procedure, we have formulated criteria derived from the plan of an experienced surgeon. The proposed system is hoped to increase the usefulness of surgical navigation systems or surgical robotics systems, as the proposed method realizes the automated preoperative planning based on objective criteria without increasing the workload of the surgeon. The present study considered the automated planning procedure of the stem. In particular, we used the cementless stem, which is fixed to the host bone by press fitting and postoperative regeneration of the bone texture. The "fit and fill" of the stem in the femoral canal are thus important factors for component stability ${ }^{(3),(4),(14)-(19)}$.

In a previous study, we defined an objective function that describes the "fitness" value of the stem based on the expertise of the surgeon and their planning results. We determined the solution using a one-by-one search at the interval to some extent in each axis ${ }^{(10),(12)}$. However, this search procedure is inappropriate because this method cannot guarantee optimal stem fitness. In addition, the constraints of position and orientation were not derived from the previous study, which represented appropriate positional relationships of the stem and femur. In contrast, Viceconti et al. proposed the use of volume registration between the femoral canal and stem for automated placement of the stem $^{(20)}$. However, the image -matching criteria used in volume registration were not derived from the stem fitness criteria used by an experienced surgeon. Position results for this method may thus be incompatible with the femoral canal of the host bone.

The present study proposes an automated planning procedure of stem placement that satisfies appropriate positional relationships of the stem and femur and guarantees optimality of stem fitness. The definition of "fitness" was as described in previous studies. In addition to this definition, we propose new constraints to describe the expertise of the surgeon regarding positional relationships of the stem and femur, and the optimization procedure to maximize fitness. to evaluate performance of the proposed system, applying the proposed method to 17 cases and measuring errors of position, orientation, and size selection between automated planning results and those of an experienced surgeon. In addition, we adjusted the parameters of the evaluate function from an exhaustive experiment using various parameters and discuss the relationship between parameters and the automated planning results.

\section{Methods}

\subsection{Overview}

A block diagram of the proposed system is shown in Fig. 1. As patient information, we assumed that a 3D-CT image of the hip joint is used for the patient. A 3D model of the femoral canal is reconstructed from CT images, and the femoral coordinate system and anatomical feature points are determined on 3D models. As implant information, we assumed that 3D shape models of all variations in the femoral stems are given. The output of this system was the set of parameters $(\mathbf{z}=[\mathbf{t}, \mathbf{r}, s])$, for the position $\mathbf{t}$, orientation $\mathbf{r}$, and size $s$ of the stem. In our formulation, we treat the parameters of size $s$, position $\mathbf{t}$, and orientation $\mathbf{r}$ independently. First, we obtain candidates for position $\mathbf{t}$ and orientation $\mathbf{r}$ in each stem size, then we select the stem size $s$.

The position and orientation of the stem are represented in the CT coordinate system. Its origin is defined as the front-left-upper corner, and the $x, y$ and $z$-axes as the left-right, front-back, and upper-lower directions, respectively.

The femoral coordinate system is shown in Fig. 2. In the specification of femoral coordinate system, we use the table-top plane of the femur and the peak of the lesser trochanter. The $z$-axis of the femoral coordinate system is defined as the canal long axis estimated from 
the 3D model of the femoral canal. The canal long axis is defined as the line connecting two points, which are the gravity center points of two different axial cross-sectional canal shapes. The distance of the two axial plane is $130 \mathrm{~mm}$ along the $z$-axis of the CT coordinate system, and the $z$-coordinate of the upper plane is added by $30 \mathrm{~mm}$ to that of the lesser tronchanter peak. The $x$-axis is orthogonal to the $z$-axis and parallel to the table-top plane. The $y$-axis is defined as the axis orthogonal to both $x$ and $z$-axes. The origin is defined as the point of intersection of the $z$-axis and a line perpendicular to the $z$-axis that passes through the peak of the lesser trochanter (Fig. 2, sagittal view).

\subsection{Definition of objective function of stem fitness}

We formulated preoperative planning of the stem as an optimization problem to obtain parameters $\mathbf{z}$ maximizing the stem fitness evaluate function. The criteria in stem planning are defined as follows:

(1) Overlap of the femoral canal and stem surface is prohibited for the entire stem area.

(2) Strong contact with the host bone in the specific area of the stem is desirable.

( 3 ) Maintain positional and rotational relationships with the femur.

(4) Select the largest stem size unless the stem is not overlapped with of the canal.

In the proposed method, we used distance from the femoral canal and stem surface to describe these criteria. A distance obtained as a negative value indicates overlap of the femoral canal and stem surface. Conversely, a distance obtained as a positive value indicates a gap between the canal and stem surface. In particular, when the gap is small, we considered strong fixation to exist. At the same time, we defined the positional and rotational constraints derived from anatomical compatibility between the host bone and stem other than stem fitness.

In our formulation, the position $\mathbf{t}$ and rotation $\mathbf{r}$ of the stem are described as the $4 \times 4$ rigid transformation matrix $\mathbf{T}$. In addition, $\mathbf{t}$ and $\mathbf{r}$ are collectively described as a six-parameter vector $\mathbf{q}=\left[t_{x}, t_{y}, t_{z}, r_{x}, r_{y}, r_{z}\right]$. We determine the parameters $\mathbf{T}$ in stem size $s$, maximizing the objective function of stem fitness $F(\mathbf{T})$, and then select the maximum stem size. The derivation of the optimal $\mathbf{T}$ is defined as the following optimization problem:

$$
\begin{array}{lll}
\operatorname{maximize}: & F(\mathbf{T})=\int_{S} f( \pm|C-\mathbf{T} \mathbf{x}|) d \mathbf{S}, \quad(\mathbf{x}=[x, y, z]) \\
\text { subject to }: & \pm|C-\mathbf{T} \mathbf{x}| \geq 0, \forall \mathbf{x} \\
& \mathbf{q}_{0}-\mathbf{q}_{r} \leq \mathbf{q} \leq \mathbf{q}_{0}+\mathbf{q}_{r}
\end{array}
$$

where $S$ describes the stem surface, and $C$ describes the femoral canal. $\mathbf{x}$ are the coordinates of the stem surface point, and $\mathbf{T} \mathbf{x}$ are the coordinates of $\mathbf{x}$ when position and orientation of the stem are $\mathbf{T} .|C-\mathbf{T x}|$ represents the distance between $\mathbf{T x}$ and the closest point on $C$ from Tx. The sign of the distance represents whether $\mathbf{T x}$ is inside or outside of $C$. When $\mathbf{T x}$ is positioned inside (the side of the femoral internal region ) of $C$, this sign is positive. Conversely, when $\mathbf{T x}$ is positioned outside (the side of the femoral host bone) of $C$, the sign is negative. The first expression of the constraints describes the constraints prohibiting overlap of the entire stem area. $\mathbf{q}_{0}$ is the central position and rotation of the range of limitation, and the range of limitation is described as $\mathbf{q}_{r}$.

To define the function $f$, we divide the stem surface into three regions. The proximal region of the surface is called zone 1, and the distal region is zone 2 (Fig. 3). Zone 1 is a specific area in which strong contact with the host bone is required. Neither zone 1 nor zone 2 can overlap with the femoral canal. However, other parts of the stem surface are not evaluated. In terms of objective function, fitness value is maximized when the gap is smaller than the constant value, and if the gap is larger than the constant, fitness value decreases with distance. The objective function is defined as (Fig. 4)

$$
f(d)=\left\{\begin{array}{ll}
1 & \left(0 \leq d<c_{t h}\right) \\
-\exp \left\{-\frac{\left(d-c_{t h}\right)^{2}}{2 \sigma^{2}}\right\} & \left(c_{t h} \leq d\right)
\end{array}, \quad(d= \pm|C-\mathbf{T x}|)\right.
$$

where $c_{t h}$ and $\sigma$ are the parameters to be adjusted. $c_{t h}$ is the threshold of the distance for which 


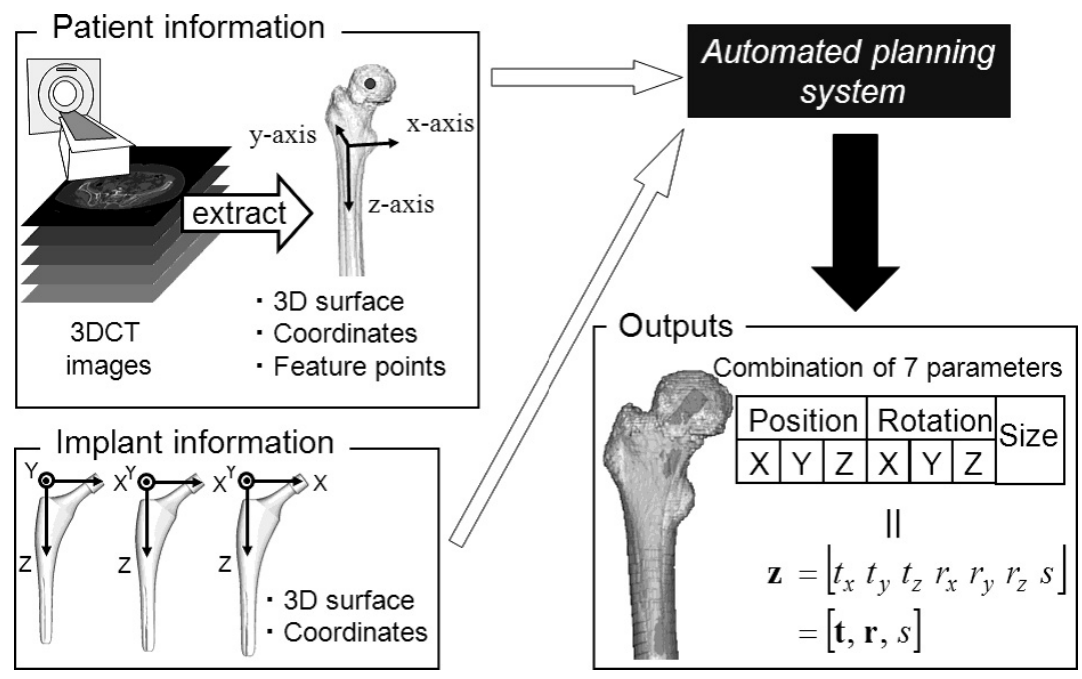

Fig. 1 Block diagram of the proposed system.

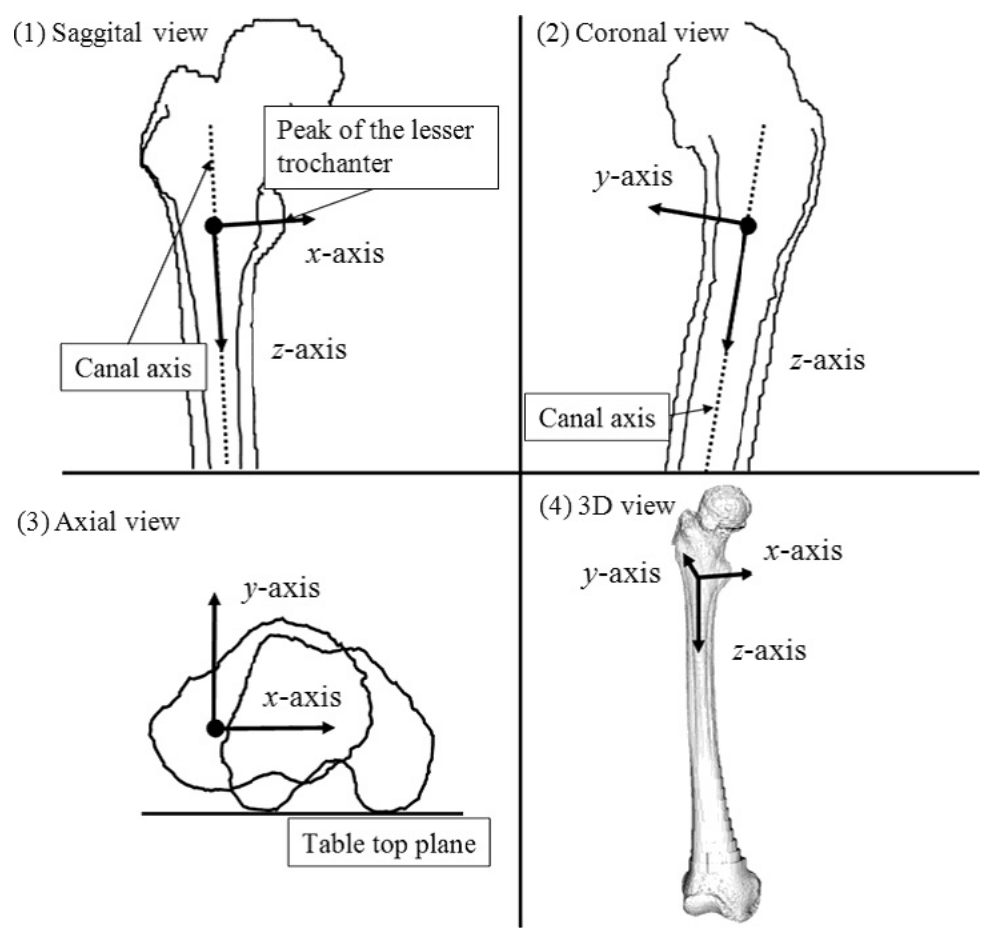

Fig. 2 Definition of the femoral coordinate system ((1) Sagittal view, (2) Coronal view, (3) Axial view, (4) 3D view)).

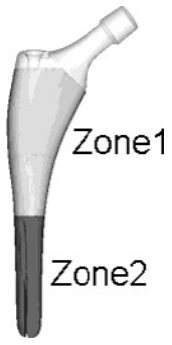

Fig. 3 Evaluation areas.

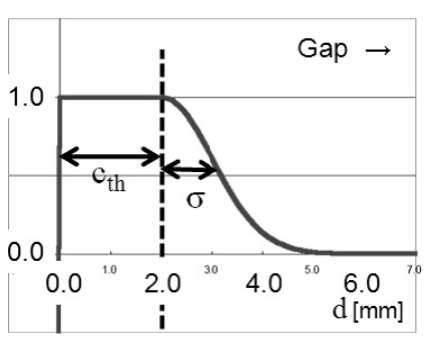

Fig. 4 Definition of $f(d)$ of stem fitness. 
the fitness value is maximized, and $\sigma$ is the parameter of the Gaussian function that describes the decrease in fitness if the distance is larger than $c_{t h}$.

\subsection{Definition of constraints}

The positional and rotational constraints used in stem planning are height of the stem position and anteversion angle. Height of the stem position affects differences in limb length during, before, and after surgery. This change in the height should be small. The change in height is defined as the difference between height of the femoral head center and height of the tip of the stem neck. The anteversion angle is the angle of the femoral neck with respect to the body of the femur (left side of Fig. 6). This angle affects the range of motion (ROM) of the hip joint After surgery, the femoral neck is removed and replaced by the stem neck. Anteversion angle after surgery is thus redefined using the stem neck (right side of the Fig. 6). This change in angle should be minimal.

To define constraint parameters $\mathbf{q}_{0}$ and $\mathbf{q}_{r}$ of the Eq. (1), we refer to conventional study of the prediction of height of the femoral head center and anteversion angle. In prediction of the femoral head center, applying sphere fitting to the deformed femoral head, as in the present study, is not appropriate. We therefore used height of the femoral neck saddle instead and limited the range to $8.3 \mathrm{~mm}$ of height difference from the femoral neck saddle based on a conventional study (Fig. 5) ${ }^{(21)}$. The height constraint is defined based on the femoral coordinate system. Anteversion angle is defined as the angle of the femoral neck axis with respect to the table-top plane (Fig. 6) ${ }^{(22)}$. Femoral neck axis is defined as the line connecting the femoral head center with the origin of the femoral coordinate system, which is projected onto the $x z$-plane of the femoral coordinate system. We fix this angle during the automated planning because we have already confirmed experimentally that the stem fitness value has no obvious peak along this parameter.

In the proposed method, the initial position $\mathbf{q}_{0}$ is defined such that the height agrees with that of the stem neck and the anteversion angle agrees with that before surgery. In our definition of coordinate axes, height corresponds to the translation along the $z$ axis, and anteversion angle corresponds to rotation around the $z$ axis. Using the criteria of the range of limitation in each axis around $\mathbf{q}_{0}, \mathbf{q}_{r}$ is defined as

$$
\mathbf{q}_{r}=[\infty, \infty, 8.3[\mathrm{~mm}], \infty, \infty, 0]^{T}
$$

\subsection{Optimization procedure}

Derivation of the candidate in each stem size is performed using the Powell method with initial values sampled within the possible solution space. We define the range of initial positions using $\mathbf{q}_{0}$ as

$$
\mathbf{q}_{0}-\mathbf{q}_{s} \leq \mathbf{q}_{I} \leq \mathbf{q}_{0}+\mathbf{q}_{s}, \quad \mathbf{q}_{s}=\left[P_{t}, P_{t}, P_{t}, P_{r}, P_{r}, 0\right]^{T}
$$

where $\mathbf{q}_{I}$ is the set of initial positions, and $P_{t}$ and $P_{r}$ are the constants that describe the range of the initial position. In the proposed method, we define these values as $P_{t}=8.0 \mathrm{~mm}$ and $P_{r}=4.0 \mathrm{deg}$. This range lies inside the constraints, and has already been confirmed experimentally to include the appropriate position and sufficient rotation. $\mathbf{q}_{I}$ takes a discrete positions, which are sampled with a fixed interval within the solution space defined in Eq. (4). The interval of the initial position $\Delta P$ is the parameter to be adjusted. We obtain a solution for each stem size using this optimization. When the optimized fitness values in each initial position are less than zero, we define this stem size as having no solution. After determining the solution for all stem sizes, we obtain stem size as the largest of all sizes displaying a solution.

\section{Experimental results}

\subsection{Experimental procedure}

We applied this method to 17 cases. First, we adjusted the parameters of the proposed 


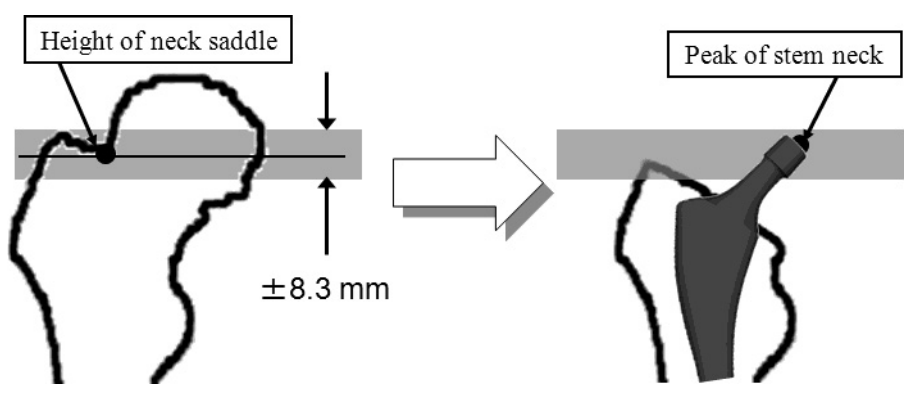

Fig. 5 Constraints: Limitation of height of the femoral neck saddle and tip of the stem neck.

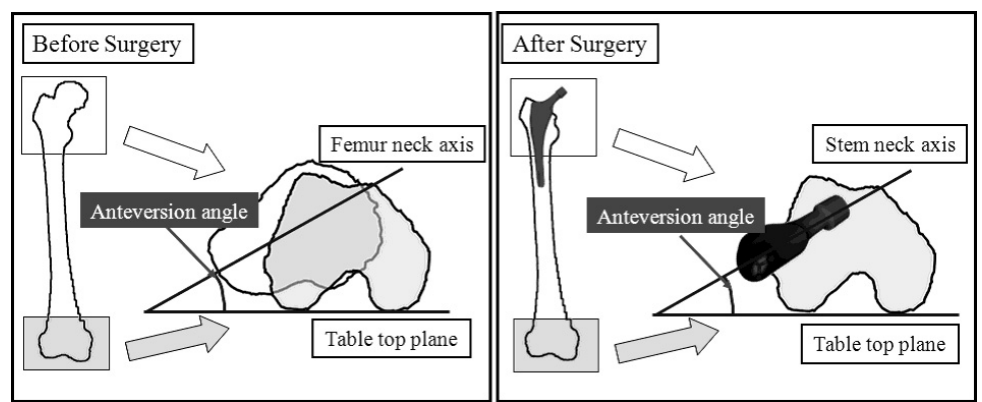

Fig. 6 Constraints: Agreement of "anteversion angle".

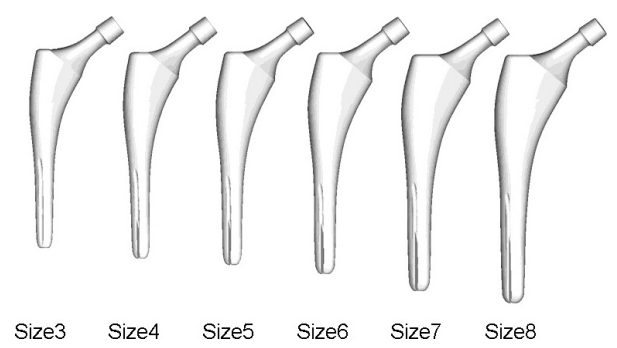

Fig. 7 Centpillar GB HA Stem (Sizes $3 \sim 8$ )

method according to an exhaustive experiment. We then compared automated plans obtained using the fine-tuned parameters and those proposed by an experienced surgeon. Planning results were evaluated from a surgeon as acceptable or not. The surgeon who assessed the plan was not the same surgeon who proposed the reference plan. Parameters of the proposed method are $c_{t h}$ and $\sigma$ in the evaluation function Eq. (2), and sample rate of the initial position is $\Delta P$ in Eq. (4). We have confirmed experimentally that the parameters in Eqs. (2) and (4) are independent of each other. We therefore adjusted the parameters of Eqs. (2) and (4) independently. The planning results were evaluated based on their differences from the surgeons' plans. The error was defined as the difference in position, orientation, and size between the automated and surgeons' plans. We compared the position and orientation of the solution for the same size as used in the surgeon's plan, although stem size selected in automated planning differed from stem size selected by the surgeon, as position and orientation change when stem size changes. The values of parameters used in the experiment were as follows:

- $\Delta P=1.0,2.0,4.0,8.0[\mathrm{~mm}]$ or [deg.]

- $c_{t h}=0.0,2.0,4.0,6.0,8.0[\mathrm{~mm}]$

- $\sigma=0.5,1.0,2.0,4.0,8.0[\mathrm{~mm}]$

For parameter tuning of the four patterns of $\Delta P$, the other parameters were set as $c_{t h}=0.0$, $\sigma=1$.0. For parameter tuning of $c_{t h}$ and $\sigma$, we used $5 \times 5=25$ patterns and $\Delta P=4.0$. 


\subsection{Experimental conditions}

We used 3D-CT images with a slice thickness of $2 \mathrm{~mm}$ (proximal slice) and $6 \mathrm{~mm}$ (distal slice). Field of view (FOV) was $360 \mathrm{~mm} \times 360 \mathrm{~mm}$. Segmentation of the 3D model of the femoral surface and femoral canal were basically performed with the threshold-based contour definition. Threshold values used to segment the femoral surface and femoral canal were 200 Hounsfield units (HU) and $800 \mathrm{HU}$, respectively. However, when appropriate segmentation was not possible due to ankylosis of the pelvis and femoral head caused by dysplasia of the hip, we used the automated segmentation system based on the statistical shape model (SSM) proposed by Okada et al. ${ }^{(23)}$. We used Centpillar GB HA Stems (Stryker Orthopaedics, Mahwah, NJ, USA) of sizes 3 to 8 (Fig. 7), the plans for which were made by experienced surgeons using the Stryker CT-Hip System (Stryker Leibinger GmbH \& Co. KG, Freiburg, Germany). In these cases, surgery was performed according to the guidance of the surgical navigation system based on the plan.

\subsection{Adjustment of parameters}

In the parameter adjustment of $\Delta P$, when $\Delta P=8.0$, the system failed to find a solution for the same stem size as the surgeon's plan in three cases. Conversely, the system succeeded in finding a solution for the same stem size as the surgeon's plan in all cases when $\Delta P=$ $1.0,2.0,4.0$. Table 1 shows the time in each $\Delta P$ for the calculation of the candidate of the one stem size (including I/O time). The calculation time of $\Delta P=1.0$ was $1,560 \mathrm{~min}$, far greater than the calculation times of the other sizes. The top left panel of Fig. 8 shows the mean error $( \pm \mathrm{SD})$ of position and orientation in each $\Delta P$. Mean errors of position and orientation were approximately the same (approximately $3.4 \mathrm{~mm}$ and 5.0 deg., respectively) for $\Delta P=1.0$ and 2.0. Standard deviations of position and orientation were also approximately the same (approximately $1.6 \mathrm{~mm}$ and $2.8 \mathrm{deg}$., respectively) for $\Delta P=1.0$ and 2.0. When $\Delta P=4.0$, mean errors of position and orientation were $3.7 \mathrm{~mm}$ and $5.2 \mathrm{deg}$., respectively, and standard deviations of position and orientation were $2.9 \mathrm{~mm}$ and $2.8 \mathrm{deg}$., respectively. Although mean errors were approximately the same as those for $\Delta P=1.0$ and 2.0, standard deviation of the positional error was larger than those for $\Delta P=1.0$ and 2.0.

In the parameter adjustment of $c_{t h}$ and $\sigma$, calculation time of optimization for the entire stem size, which was approximately the same in all conditions, was approximately $20 \mathrm{~min}$. The top right panel of Fig. 8 shows the number of cases in which the difference in stem size from the surgeon's plan was less than one size. When $c_{t h} \leq 2.0$ and $\sigma \leq 2.0$, differences in stem size selection were less than one size in all cases. The bottom of Fig. 8 shows mean errors of position in each $c_{t h}$ and $\sigma$. Mean positional errors increased when $c_{t h} \geq 6.0$. On the other hand, the positional error changed little and the error was small when $c_{t h}<6.0$ except for $c_{t h}=0.0, \sigma=0.5$. The smallest positional mean error, $3.7 \mathrm{~mm}$, was attained when $c_{t h}=1.0$ and $\sigma=1.0$. From the results of orientation, mean errors were approximately 5.3 deg. in all conditions. Few changes were seen with changes of parameters.

\subsection{Results of automated planning with fine-tuned parameters}

From the results of the adjustment of parameters, we set the parameters as $\Delta P=2.0, c_{t h}=$ 0.0 , and $\sigma=1.0$. Calculation time to find the solution in entire stem size was approximately $360 \mathrm{~min}$ in each case. The difference in stem size was less than one size from the surgeon's plan in all cases. According to the assessment of the surgeon, 14 cases were accepted and three cases were rejected. Figure 9 shows mean errors of position and orientation in each coordinate axis of the groups of accepted and rejected cases. Mean error of position was 3.2 $\mathrm{mm}$ in accepted cases and $4.0 \mathrm{~mm}$ in rejected cases, and that of orientation was $4.6 \mathrm{deg}$. in accepted cases and $7.0 \mathrm{deg}$. in rejected cases. The difference between accepted and rejected cases in mean error of orientation was larger than that of the mean error of position. In particular, mean error of orientation in $z$-axis, which was assigned as the anteversion angle of the femur and stem neck, differed between accepted and rejected cases. 
Table 1 Result of parameter adjustment: calculation time in each $\Delta P$ [min]

\begin{tabular}{|l|r|}
\hline & Calculation time [min] \\
\hline$\Delta \mathrm{P}=8.0$ & 0.5 \\
\hline$\Delta \mathrm{P}=4.0$ & 5 \\
\hline$\Delta \mathrm{P}=2.0$ & 75 \\
\hline$\Delta \mathrm{P}=1.0$ & 1560 \\
\hline
\end{tabular}
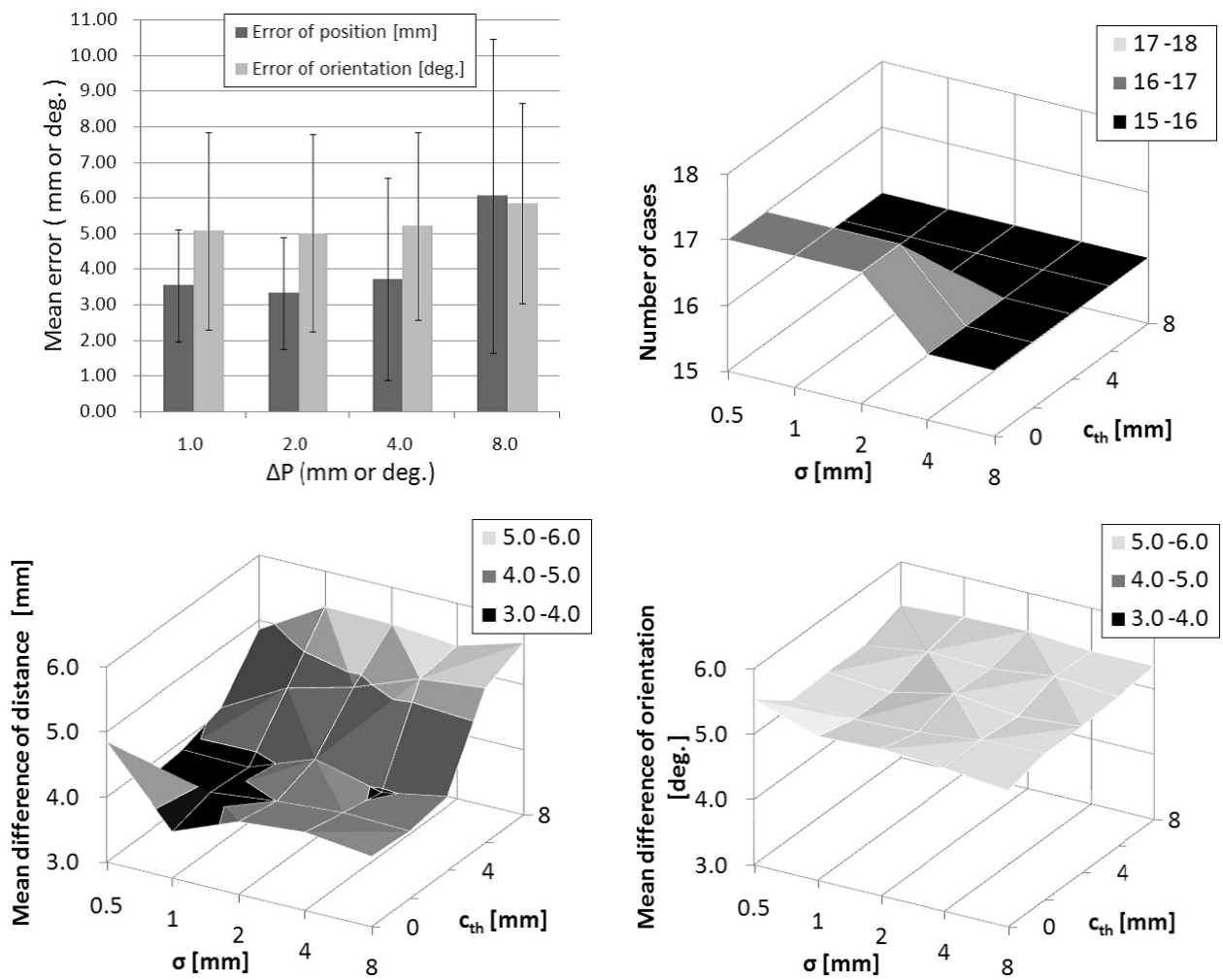

Fig. 8 Results of parameter adjustment. Top left: mean error $( \pm$ SD) of position and orientation in each $\Delta P$. Top right: number of cases in which the difference in size on automated planning is less than 1 size. Bottom left and bottom right: mean difference of position and mean difference of orientation, respectively, for the proposed method.

Figures 10 and 11 show the visualization of planning results by the experienced surgeon and the automated system for one of the accepted cases (Case 5) and one of the rejected cases (Case 1). The color map on the stem surface indicates distance between the stem surface and femoral canal, which was used in the calculation of stem fitness. In the distribution of distance, yellow and green areas are considered areas of strong stem fitness. In Case 5, positional error of the automated plan was $0.5 \mathrm{~mm}$, and orientational error was $3.4 \mathrm{deg}$. Conversely, in Case 1, positional error was $5.5 \mathrm{~mm}$, and orientational error was $9.2 \mathrm{deg}$. In particular, orientational error of the $z$-axis was $8.9 \mathrm{deg}$., revealing a significant difference from the planning results (right figure in each condition of Fig. 11).

\section{Discussions}

We have developed an automated planning system to properly determine the size, position, and orientation of each implant by maximizing stem fitness. We then adjusted parameters of the criteria for the proposed method to apply this method to the Centpillar GB HA stem.

We discuss the parameter adjustment of $\Delta P, c_{t h}$, and $\sigma . \Delta P$ is the sample rate of initial positions in the optimization. For $\Delta P=8.0$, in three cases, the automated system failed to find a solution for the same stem size as that used in the surgeon's plan. In addition, mean errors were larger than those of the other conditions of $\Delta P$. As a result, $\Delta P$ is thought to affect the precision of optimization. $\Delta P$ also affects calculation time, as local optimization is 


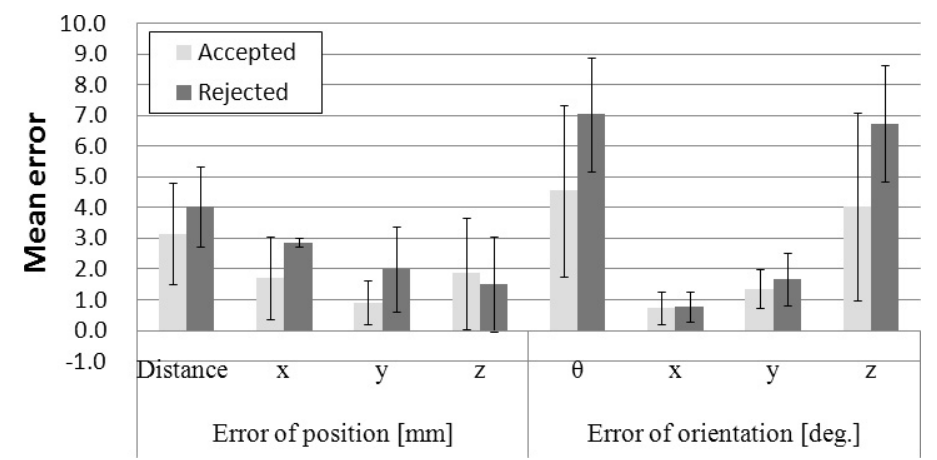

Fig. 9 Mean error of automated planning with fine-tuned parameters of accepted and rejected cases as assessed by a surgeon.

performed cyclopedically in the solution space $\mathbf{q}_{I}$, which is sampled by $\Delta P$. In comparing the calculation times of results for $\Delta P=1.0$ and 2.0, $\Delta P=1.0$ required a calculation time 22 times longer than that for $\Delta P=2.0$, although mean errors of position and orientation were approximately the same. Based on these results, we consider $\Delta P=2.0$ as the balanced value of this parameter. $c_{t h}$ and $\sigma$ represent the relationship between distance between the femoral canal and stem surface and stem fitness, respectively. As these parameters increase, latitude of the gap between the stem surface and femoral canal also increases. When $c_{t h} \geq 4.0$ or $\sigma \geq 4.0$, the automated system failed to find a solution for the appropriate stem size. Positional error was also large under these conditions. Conversely, although $c_{t h}<4.0$ and $\sigma<4.0$, positional error was large when $c_{t h}=0.0$ and $\sigma=0.5$. Positional errors of the rest were almost the same. The results indicate that the ranges of parameters for obtaining reasonable plans are $0.0 \leq c_{t h} \leq 4.0$ and $1.0 \leq \sigma \leq 2.0$. We determined $c_{t h}=0.0$ and $\sigma=1.0$ after taking into account the design principle of the stem that strong fixation with no gap is required.

We discuss the performance of the proposed system based on the automated planning results obtained with the fine-tuned parameters. In stem size selection, the difference between the automated system and the experienced surgeon was less than 1 size in all cases. This result appears acceptable, as stem selection varies in size to some extent because of limb length adjustments. In addition, the surgeon accepted the automated plan in 14 cases. The remaining three cases were acceptable after slight readjustment by surgeon. Given these results, the proposed method appears applicable as an automated preoperative planning system. The remaining problem that appeared in rejected cases was performance of the determination of orientation. The mean error of orientation in cases rejected by the surgeon was $7.0 \mathrm{deg}$. This is thought to have been due to insufficient description of the surgeon's expertise. In the proposed method, orientation along the $z$-axis, which was assigned with the angle of anteversion, was fixed as the original femoral anteversion by the constraints (described in §2.3). However, the surgeon often does not use this criterion in adjusting the angle, considering the fitness in the stem neck area. To overcome this problem, we will define the fitness in the stem neck and estimation of the orientation of the $z$-axis.

In the proposed fitness function, the small gaps between the stem and canal surfaces are allowable. This criterion is based on the clinical knowledge that the small gaps will be filled due to bone regrowth so that sufficient fixation is attained. Therefore, we do not consider that these small gaps cause problems. In addition, we did not precisely adjust the area for contact evaluation within Zone 1 in Fig. 3. In our preliminary experiments, we compared the results of between several patterns of specific areas and the whole area of Zone 1, and significant differences were not observed as long as the Centpillar stem was used. Thus, we did not precisely adjust the evaluation area.

In general, segmentation requires a great deal of work, since the user must segment the region frame-by-frame in CT images. About one hour is required for this method for manual 


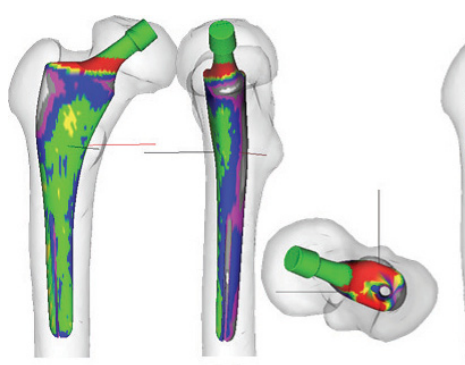

(a)

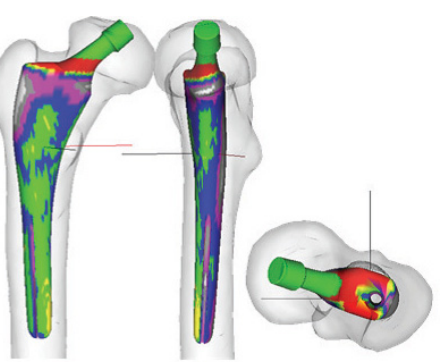

(b)

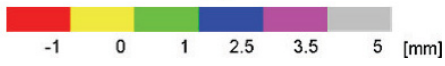

Fig. 10 Visualization of planning results for an accepted case (Case 5). ((a) Surgeon's plan, (b) Automated plan).

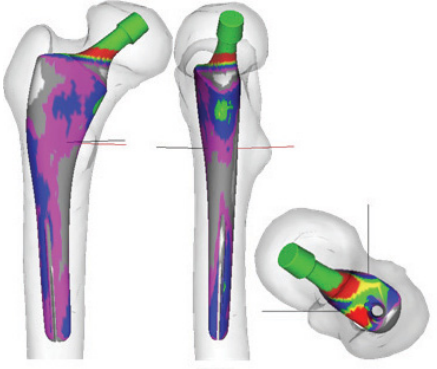

(a)

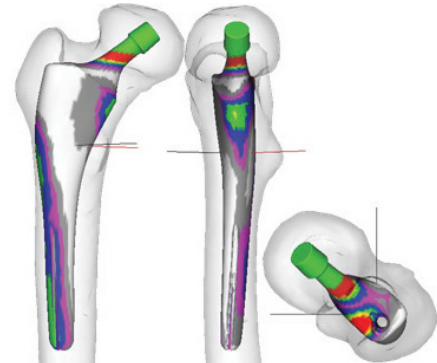

(b)

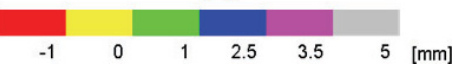

Fig. 11 Visualization of planning results from a rejected case (Case 1). ((a) Surgeon's plan, (b) Automated plan).

segmentation of the femur and femoral canal. In the proposed system, we employed the automated segmentation procedure of the 3D femur and femoral canal shapes proposed by Okada et al. ${ }^{(23)}$ to overcome this problem. This automated segmentation procedure uses the SSM, which describes the statistical trend of the shape constructed from manually segmented shape models. Using this method, the manual operation time of segmentation decreases to 5 min for obtaining anatomical feature points. Since segmentation is performed automatically based on several anatomical feature points on the femur, the workload for the surgeon is small. We thus do not consider segmentation of the femur and femoral canal region as an obstacle to clinical use.

The proposed method takes approximately 360 min for automated planning in the current implementation. Although approximately $360 \mathrm{~min}$ is required for automated planning, computational time can be reduced if parallel processing is introduced. Since automated planning procedures are performed without any interaction, the resulting plan can be obtained overnight if input data are provided. Calculation time would thus be acceptable because the surgeon only needs to perform several minutes of work, in identifying some anatomical features on the femur.

\section{Conclusions}

We have developed an automated planning system of the stem for THA. By executing exhaustive parameter tuning of the objective function, we succeeded in achieving planning results similar to the plans prepared by an experienced surgeon. We believe that the workload of the surgeon can be reduced in preoperative planning. In addition, even inexperienced surgeons can obtain appropriate plans close to that of an experienced surgeon by using this system. The proposed system would thus increase the usefulness of various intraoperative surgery systems, such as surgical navigation and robotics systems, One problem in the proposed method that 
remains to be solved is that parameter tuning must be performed when other stem products are applied to this system, as the pair of parameter values depends on stem shape, and other stem products may differ in shape.

In the future, we will incorporate criteria for mechanical analysis and the evaluation function of stem fitness without adjustment of parameters. In a mechanical analysis of stem planning, Reggiani et al. ${ }^{(24)}$, as well as other researchers, reported the distribution of stress from the stem for specific conditions using a finite element model. However, the criteria of preoperative planning to achieve appropriate distributions of stress have not yet been proposed. If these criteria are proposed, reality-based planning with more than only the one criteria of stem fitness will be possible. In addition, to define stem fitness evaluation function without adjustment of the parameter, we will apply a method based on statistical analysis of the surgeon's plans.

\section{Acknowledgement}

This research was supported in part by Stryker Japan K. K. and Med-Solution, Inc.

\section{References}

( 1 ) Sugano, N., Computer-assisted orthopedic surgery, Journal of Orthopaedic Science, Vol.8, No.3 (2003), pp.442-448.

( 2 ) Bargar, W. L., Bauer, A., Borner, M., Primary and revision total hip replacement using the Robodoc system, Clinical Orthopaedics $\mathcal{E}$ Related Research, Vol.354 (1998), pp.8291.

( 3 ) Haraguchi, K., Sugano, N., Nishi, T., Koyama, T., Nishihara, S., Yoshikawa, H., Ochi, T., Comparison of fit and fill between anatomic stem and straight tapered stem using virtual implantation on the ORTHODOC workstation, Computer aided surgery : official journal of the International Society for Computer Aided Surgery, Vol.6, No.5 (2001), pp. 290-296.

( 4 ) Nishihara, S., Sugano, N., Nishii, T., Miki, H., Nakamura, N., Yoshikawa, H., Comparison Between Hand Rasping and Robotic Milling for Stem Implantation in Cementless Total Hip Arthroplasty, The Journal of Arthroplasty, Vol.21, Issue 7 (2006), pp. 957-966.

( 5 ) Digioia III, A. M., Jaramaz, B., Nikou, C., Labarca, R. S., Moody, J. E., Colgan, B. D., Surgical navigation for total hip replacement with the use of hipnav, Operative Techniques in Orthopaedics, Vol.10, Issue 1 (2000), pp. 3-8.

( 6 ) Honl, M., Dierk, O., Gauck, C., Carrero, V., Lampe, F., Dries, S., Quante, M., Schwieger, K., Hille, E., Morlock, M.M., Comparison of robotic-assisted and manual implantation of a primary total hip replacement: A prospective study, Journal of Bone and Joint Surgery - Series A, Vol. 85, Issue 8 (2003), pp. 1470 -1478.

( 7 ) Ecker, T.M., Tannast, M., Murphy, S.B., Computed tomography-based surgical navigation for hip arthroplasty, Clinical Orthopaedics and Related Research, Issue 465 (2007), pp. $100-105$.

( 8 ) Lattanzi, R., Viceconti, M., Zannoni, C., Quadrani, P., Toni, A., Hip-Op: an innovative software to plan total hip replacement surgery, Medical Informatics and the Internet in Medicine, Vol.27, No.2 (2002), pp. 71-83.

( 9 ) Testi, D., Simeoni, M., Zannoni, C., and Viceconti, M., Validation of two algorithms to evaluate the interface between bone and orthopaedic implants, Computer Methods and Programs in Biomedicine, Vol.74, Issue 2 (2004), pp. 143.150.

(10) Nakamoto, M., Sato, Y., Sugano, N., Sasama, T., Nishii, T., Pak, P. S., Akazawa, K., Tada, Y., Yoshikawa, H., Tamura, S., Automated CT-based 3D surgical planning for total hip replacement: a pilot study,International Congress Series, Vol.1256 (2003), pp. 389-394.

(11) Kagiyama, Y., Nakamoto, M., Takao, M., Sato, Y., Sugano, N., Tada, Y., Automated preoperative 3D planning for multi-component implants based on leg length evalua- 
tion in total hip arthroplasty using CT data: pilot study, 5th Annual Meeting of CAOSInternational Proceedings (2005), pp. 204-206.

(12) Otomaru, I., Takao, M., Nakamoto, M., Sugano, N., Kagiyama, Y., Sato, Y., Automated Preoperative Planning System of Total Hip Arthroplasty Using Anatomical Femoral Components, 6th Annual Meeting of CAOS-International Proceedings (2006), pp. 419423.

(13) Kagiyama, Y., Takao, M., Nakamoto, M., Sato, Y. Sugano, N., Yoshikawa, H., Akazawa, K., Tamura, S., Tada, Y. Automated planning of multi-component implants for THA using multiple criteria including limb length and range of motion, International Journal of Computer Assisted Radiology and Surgery, Vol. 1, Supplement 1 (2006), pp. 232-234.

(14) Bargar, W. L., Shape the Implant to the Patient. A Rationale for the Use of Custom-Fit Cementless Total Hip Implants, Clinical orthopaedics and related research, Vol. 249 (1989), pp. 73-78.

(15) Bo, A., Imura, S., Omori, H., Okumura, Y., Ando, M., Baba, H., White, P., Zarnowski, A., Fit and fill analysis of a newly designed femoral stem in cementless total hip arthroplasty for patients with secondary osteoarthritis, Journal of Orthopaedic Science, Vol.2, No.5 (1997), pp. 301-312.

(16) Nishihara, S., Sugano N., Nishii, T., Tanaka H., Yoshikawa, H., Ochi, A., Comparison of the fit and fill between the Anatomic Hip femoral component and the VerSys Taper femoral component using virtual implantation on the ORTHODOC workstation, Journal of Orthopaedic Science, Vol.8, No.3 (2003), pp. 352-360.

(17) Howard, J. L., Hui, A. J., Bourne, R. B., McCalden, R. W., MacDonald, S. J., Rorabeck, C. H., A quantitative analysis of bone support comparing cementless tapered and distal fixation total hip replacements, The Journal of Arthroplasty, Vol.19, Issue 3 (2004), pp. 266-273.

(18) Laine, H. J., Puolakka, T. J. S., Moilanen, T., Pajamaki, K. J., Wirta, J., Lehto, M. U. K., The effects of cementless femoral stem shape and proximal surface texture on "fit-andfill" characteristics and on bone remodeling, International Orthopaedics, Vol.24, No.4 (2000), pp. 184-190.

(19) Laine, H. J., Pajamaki, J. K., Moilanen, T., Lehto, M. U., The femoral canal fill of two different cementless stem designs: The accuracy of radiographs compared to computed tomographic scanning, International Orthopaedics, Vol.25, No.4 (2001), pp. 209-213.

(20) Viceconti, M., Testi, D., Simeoni, M., Zannoni, C., An automated method to position prosthetic components within multiple anatomical spaces,Computer Methods and Programs in Biomedicine, Vol.70, Issue 2 (2003), pp. 121-127.

(21) Sugano, N., Noble, P. C., Kamaric, E., Predicting the Position of the Femoral Head Center, The Journal of Arthroplasty, Vol.14, No. 1 (1999), pp. 102-107.

(22) Sugano, N., Noble, P. C., Kamaric, E., A comparison of alternative methods of measuring femoral anteversion, Journal of Computer Assisted Tomography, Vol.22, Issue 4 (1998), pp. 610-614.

(23) Okada, T., Shimada, R., Sato, Y., Hori, M., Yokota, K., Nakamoto M., Chen, Y., Nakamura, H., Tamura, S., Automated Segmentation of the Liver from 3D CT Images Using Probabilistic Atlas and Multi-level Statistical Shape Model, Medical Image Computing and Computer-Assisted Intervention - MICCAI 2007, Vol. 4791 (2007), pp. 86-93.

(24) Reggiani, B., Cristofolini, L., Varini, E., Viceconti, M., Predicting the subject-specific primary stability of cementless implants during pre-operative planning: Preliminary validation of subject-specific finite-element models, Journal of Biomechanics, Vol. 40, Issue 11 (2007), pp. 2552-2558. 